\title{
First and second fundamental solutions of the time-fractional telegraph equation with Laplace or Dirac operators*
}

\author{
M. Ferreira ${ }^{\dagger, \ddagger} \quad$ M.M. Rodrigues ${ }^{\ddagger} \quad$ N. Vieira ${ }^{\ddagger}$ \\ $\dagger$ School of Technology and Management, \\ Polytechnic Institute of Leiria \\ P-2411-901, Leiria, Portugal. \\ $\ddagger$ CIDMA - Center for Research and Development in Mathematics and Applications \\ Department of Mathematics, University of Aveiro \\ Campus Universitário de Santiago, 3810-193 Aveiro, Portugal. \\ E-mails: milton.ferreira@ipleiria.pt, mrodrigues@ua.pt, nloureirovieira@gmail.com
}

\begin{abstract}
In this work we obtain the first and second fundamental solutions (FS) of the multidimensional timefractional equation with Laplace or Dirac operators, where the two time-fractional derivatives of orders $\alpha \in] 0,1]$ and $\beta \in] 1,2]$ are in the Caputo sense. We obtain representations of the FS in terms of Hankel transform, double Mellin-Barnes integrals, and H-functions of two variables. As an application, the FS are used to solve Cauchy problems of Laplace and Dirac type.
\end{abstract}

Keywords: Time-fractional telegraph equation; Telegraph Dirac operator; First and second fundamental solutions; Caputo fractional derivative; Multivariate Mittag-Leffler function; H-function of two variables.

MSC 2010: 30G35; 35R11, 26A33, 35A08, 35C15, 33E12.

\section{Introduction}

In the last years, the interest in the study of fractional differential equations has increased considerably due essentially to the wide range of applications. These type of equations are used mostly to model processes in the fields of engineering, viscoelastic materials, hydrology, system control, just to mention some. The use of fractional derivatives give us the possibility of describing memory and heredity properties of the processes (see $[14,16])$. However, for some fractional differential operators, the deduction of an explicit integral and/or series representations of the FS is still an open question.

The telegraph equation is used as an alternative to the diffusion equation, since it has the potential to describe both diffusive and wave-like phenomena, due to the simultaneous presence of first and second order time derivatives. For example, in the case of the transport of energetic charged particle in turbulent magnetic fields such as low-energy cosmic rays in the solar wind, the diffusion equation can not be used to describe the transport for early times because it leads to a non-zero probability density everywhere, which would correspond to an infinite propagation speed. Using the telegraph equation in this case we get a more realistic model for the early phase transport because it combines diffusion with a finite propagation speed (see [18]). Telegraph equations have also an extraordinary importance in electrodynamics (the scalar Maxwell equations are of this

*The final version is published in Advances in Applied Clifford Algebras, 28-No.2, (2018), 28-42. It as available via the website: https://link.springer.com/article/10.1007/s00006-018-0858-7 
type), in the theory of damped vibrations, and in probability because they are connected with finite velocity random motions (see $[14,16]$ ).

The aim of this paper is to obtain explicit integral representations for the first and second FS of the timefractional telegraph equation with Laplace or Dirac operators. The first case corresponds to a combination of the Laplace operator in space with the sum of two time-fractional derivatives of orders $\alpha \in] 0,1]$ and $\beta \in] 1,2]$ in the Caputo sense, while in the second case we have the Dirac operator in space combined with a sum of the two time-fractional derivatives, using a Witt basis in the framework of Clifford algebra. The second operator factorizes the first one (see [9]). Connections between Clifford analysis and fractional calculus were recently established in the study of the stationary fractional Dirac operator (see [10,11]) and in the study of the time-fractional diffusion-wave and telegraph operators (see [7,9]).

The results presented in this paper are a generalization of the results presented in $[8,9]$, since we incorporate the second fundamental solution coming from the second initial condition (see formulation in (9)).

The structure of the paper reads as follows: in the Preliminaries section we recall some basic facts about some special functions and fractional calculus, which are necessary for the development of the work. In Section 3 we obtain the FS of the time-fractional telegraph equation with Laplace operator, while in Section 4 we compute the FS for the time-fractional telegraph equation with Dirac operator. In both cases, we deduce several representations of the FS in the form of Hankel transform, double Mellin-Barnes integrals, and Hfunctions of two variables. Both sections end with applications to the resolution of Cauchy-type problems. Some connections with previous works are also presented.

\section{Preliminaries}

In this section we present the main tools concerning fractional derivatives and special functions that will be used in our work. We start by recalling the definition of the multivariate Mittag-Leffler function (see [15]).

Definition 2.1 The multivariate Mittag-Leffler function $E_{\left(a_{1}, \ldots, a_{n}\right), b}\left(z_{1}, \ldots, z_{n}\right)$ ofn complex variables $z_{1}, \ldots, z_{n} \in$ $\mathbb{C}$ with complex parameters $a_{1}, \ldots, a_{n}, b \in \mathbb{C}$ (with positive real parts) is defined by

$$
E_{\left(a_{1}, \ldots, a_{n}\right), b}\left(z_{1}, \ldots, z_{n}\right)=\sum_{k=0}^{+\infty} \sum_{\substack{l_{1}+\ldots+l_{n}=k \\
l_{1}, \ldots, l_{n} \geq 0}}\left(\begin{array}{c}
k \\
l_{1}, \ldots, l_{n}
\end{array}\right) \frac{\prod_{i=1}^{n} z_{i}^{l_{i}}}{\Gamma\left(b+\sum_{i=1}^{n} a_{i} l_{i}\right)}
$$

where the multinomial coefficients are given by

$$
\left(\begin{array}{c}
k \\
l_{1}, \ldots, l_{n}
\end{array}\right):=\frac{k !}{l_{1} ! \times \ldots \times l_{n} !} .
$$

When $n=2$ we obtain the bivariate Mittag-Leffler function, which can be written as

$$
E_{\left(a_{1}, a_{2}\right), b}\left(z_{1}, z_{2}\right)=\sum_{l_{1}=0}^{+\infty} \sum_{l_{2}=0}^{+\infty} \frac{\left(l_{1}+l_{2}\right) !}{l_{1} ! l_{2} !} \frac{z_{1}^{l_{1}} z_{2}^{l_{2}}}{\Gamma\left(b+a_{1} l_{1}+a_{2} l_{2}\right)}
$$

From (2) we can deduce, after straightforward calculations, an addition formula for the bivariate Mittag-Leffler function.

Lemma 2.2 Let $z_{1}, z_{2} \in \mathbb{C}$, and $a_{1}, a_{2}, b \in \mathbb{C}$ (with positive real parts). Then it holds

$$
E_{\left(a_{1}, a_{2}\right), b}\left(z_{1}, z_{2}\right)=\frac{1}{\Gamma(b)}+z_{1} E_{\left(a_{1}, a_{2}\right), b+a_{1}}\left(z_{1}, z_{2}\right)+z_{2} E_{\left(a_{1}, a_{2}\right), b+a_{2}}\left(z_{1}, z_{2}\right) .
$$

For general properties of the Mittag-Leffler function see [13,15]. Now we recall the definition of the H-function of two complex variables.

Definition 2.3 (see [2]) The H-function of two complex variables is defined via a double Mellin-Barnes type 
integral of the form

$$
\begin{array}{r}
H_{p_{1}, q_{1} ; p_{2}, q_{2} ; p_{3}, q_{3}}^{0, n_{1} ; m_{3}, n_{2} ; m_{3}, n_{3}}\left[\begin{array}{c|c}
x & \left(a_{j} ; \alpha_{j}, A_{j}\right)_{1, p_{1}} ;\left(c_{j}, \gamma_{j}\right)_{1, p_{2}} ;\left(e_{j}, E_{j}\right)_{1, p_{3}} \\
y & \left(b_{j} ; \beta_{j}, B_{j}\right)_{1, q_{1}} ;\left(d_{j}, \delta_{j}\right)_{1, q_{2}} ;\left(f_{j}, F_{j}\right)_{1, q_{3}}
\end{array}\right] \\
=\frac{1}{(2 \pi i)^{2}} \int_{\mathcal{L}_{2}} \int_{\mathcal{L}_{1}} \phi(s, w) \phi_{1}(s) \phi_{2}(w) x^{s} y^{w} d s d w
\end{array}
$$

where

$$
\begin{aligned}
& \phi(s, w)=\frac{\prod_{i=1}^{n_{1}} \Gamma\left(1-a_{i}+\alpha_{i} s+A_{i} w\right)}{\prod_{i=n_{1}+1}^{p_{1}} \Gamma\left(a_{i}-\alpha_{i} s-A_{i} w\right) \prod_{j=1}^{q_{1}} \Gamma\left(1-b_{j}+\beta_{j} s+B_{j} w\right)}, \\
& \phi_{1}(s)=\frac{\prod_{j=1}^{m_{2}} \Gamma\left(d_{j}-\delta_{j} s\right) \prod_{i=1}^{n_{2}} \Gamma\left(1-c_{i}+\gamma_{i} s\right)}{\prod_{j=m_{2}+1}^{q_{2}} \Gamma\left(1-d_{j}+\delta_{j} s\right) \prod_{i=n_{2}+1}^{p_{2}} \Gamma\left(c_{i}-\gamma_{i} s\right)}, \\
& \phi_{2}(w)=\frac{\prod_{j=1}^{m_{3}} \Gamma\left(f_{j}-F_{j} w\right) \prod_{i=1}^{n_{3}} \Gamma\left(1-e_{i}+E_{i} w\right)}{\prod_{j=m_{3}+1}^{q_{3}} \Gamma\left(1-f_{j}+F_{j} w\right) \prod_{i=n_{3}+1}^{p_{3}} \Gamma\left(e_{i}-E_{i} w\right)},
\end{aligned}
$$

and where an empty product is interpreted as $1, x, y \in \mathbb{C}, m_{i}, n_{i}, p_{i}, q_{i} \in \mathbb{Z}$ such that $0 \leq m_{i} \leq q_{i}, 0 \leq n_{i} \leq p_{i}$ $(i=1,2,3) ; a_{i}, b_{j}, c_{i}, d_{j}, e_{i}, f_{j} \in \mathbb{C}, \alpha_{i}, A_{i}, \beta_{j}, B_{j}, \gamma_{i}, \delta_{j}, E_{i}, F_{j} \in \mathbb{R}^{+}$and the sequence of parameters $\left(a_{j}\right),\left(b_{j}\right)$, $\left(c_{j}\right),\left(d_{j}\right),\left(e_{j}\right)$ and $\left(f_{j}\right)$ are restricted that none of the poles of the integrand coincide. The contour $\mathcal{L}_{1}$ in the complex s-plane, and the contour $\mathcal{L}_{2}$ in the complex w-plane, are of Mellin-Barnes type with indentations, if necessary, to ensure that they separate one set of poles from the other.

In [2] the author proved that if

$$
\begin{aligned}
& \Omega_{1}=\sum_{i=1}^{n_{1}} \alpha_{i}-\sum_{i=n_{1}+1}^{p_{1}} \alpha_{i}-\sum_{i=1}^{q_{1}} \beta_{i}+\sum_{i=1}^{m_{2}} \delta_{i}-\sum_{j=m_{2}+1}^{q_{2}} \delta_{i}+\sum_{i=1}^{n_{2}} \gamma_{i}-\sum_{i=n_{2}+1}^{p_{2}} \gamma_{i} \geq 0, \\
& \Omega_{2}=\sum_{i=1}^{n_{1}} A_{i}-\sum_{i=n_{1}+1}^{p_{1}} A_{i}-\sum_{i=1}^{q_{1}} B_{i}+\sum_{i=1}^{m_{3}} F_{i}-\sum_{j=m_{3}+1}^{q_{3}} F_{i}+\sum_{i=1}^{n_{3}} E_{i}-\sum_{i=n_{3}+1}^{p_{3}} E_{i} \geq 0,
\end{aligned}
$$

and with the points $x=0$ and $y=0$ being tacitly excluded, the double Mellin-Barnes integral converges absolutely inside the sector given by

$$
|\arg (x)|<\frac{\pi}{2} \Omega_{1}, \quad|\arg (y)|<\frac{\pi}{2} \Omega_{2} .
$$

When all poles of the integrand are simple, we have the following asymptotic behaviour for large values of $x$ and $y$ (see [1])

$$
H[x, y]=O\left(|x|^{\beta_{1}},|y|^{\beta_{2}}\right),
$$

where

$$
\begin{array}{ll}
\beta_{1}=\max \left\{\Re\left(\frac{c_{j}-1}{\delta_{j}}\right)\right\}, & j=1,2, \ldots, n_{2}, \\
\beta_{2}=\max \left\{\Re\left(\frac{e_{j}-1}{E_{j}}\right)\right\}, & j=1,2, \ldots, n_{3} .
\end{array}
$$

For $E_{\left(a_{1}, a_{2}\right), b}$ we have the following two results (see [8]).

Lemma 2.4 The bivariate Mittag-Leffler function $E_{\left(a_{1}, a_{2}\right), b}$ has the following representation in the form of double Mellin-Barnes integral

$$
E_{\left(a_{1}, a_{2}\right), b}\left(z_{1}, z_{2}\right)=\frac{1}{(2 \pi i)^{2}} \int_{\mathcal{L}_{1}} \int_{\mathcal{L}_{2}} \frac{\Gamma(1+s+w) \Gamma(-s) \Gamma(-w)}{\Gamma\left(b+a_{1} w+a_{2} s\right)} z_{2}^{s} z_{1}^{w} d w d s .
$$


Corollary 2.5 The bivariate Mittag-Leffler function $E_{\left(a_{1}, a_{2}\right), b}$ has the following representation in the form of Fox H-function of two variables

$$
E_{\left(a_{1}, a_{2}\right), b}\left(z_{1}, z_{2}\right)=H_{1,1 ; 0,1 ; 0,1}^{0,1 ; 1,0 ; 1,0}\left[\begin{array}{c|c}
z_{2} & (0 ; 1,1) ;-;- \\
z_{1} & \left(1-b ; a_{2}, a_{1}\right) ;(0,1) ;(0,1)
\end{array}\right]
$$

In (6) and throughout the paper a horizontal line in the H-function means the absence of parameters. Now we recall the definition of the spaces $C_{\alpha}, \alpha \in \mathbb{R}$, and $C_{\alpha}^{m}, m \in \mathbb{N}$, given in [15].

Definition 2.6 (see [15]) A real or complex-valued function $f(t), t>0$, is said to be in the space $C_{\alpha}, \alpha \in \mathbb{R}$, if there exists a real number $p>\alpha$ such that $f(t)=t^{p} f_{1}(t)$ for some function $f_{1} \in C[0, \infty)$.

It is easy to see that $C_{\alpha}$ is a vector space and the set of spaces $C_{\alpha}$ is ordered by inclusion according to $C_{\alpha} \subseteq C_{\beta} \Leftrightarrow \alpha \geq \beta$.

Definition 2.7 (see [15]) A function $f(t), t>0$, is said to be in the space $C_{\alpha}^{m}, m \in \mathbb{N}$, if and only if $f^{(m)} \in C_{\alpha}$. Let $D_{t}^{\gamma}$ denotes the Caputo fractional derivative of order $\gamma>0$ defined by:

$$
D_{t}^{\gamma} u(t)=\left\{\begin{array}{cl}
\frac{1}{\Gamma(m-\gamma)} \int_{0}^{t}(t-s)^{-\gamma+m-1} u^{(m)}(s) d s, & m-1<\gamma<m \\
u^{(m)}(t), & \gamma=m
\end{array}\right.
$$

where $u^{(m)}:=\frac{d^{m} u}{d t^{m}}, m \in \mathbb{N}$ (see [3]). The next theorem will be used in our analysis and allow us to solve general linear fractional differential equations with constant coefficients and Caputo derivatives.

Theorem 2.8 (see [15, Thm 4.1]) Let $\mu>\mu_{1}>\ldots>\mu_{n} \geq 0, m_{i}-1<\mu_{i} \leq m_{i}, m_{i} \in \mathbb{N}, \lambda_{i} \in \mathbb{R}, i=1, \ldots, n$. Consider the initial value problem

$$
\left\{\begin{array}{l}
D_{t}^{\mu} y(t)-\sum_{i=1}^{n} \lambda_{i} D_{t}^{\mu_{i}} y(t)=g(t) \\
y^{(k)}(0)=c_{k} \in \mathbb{R}, \quad k=0, \ldots, m-1, \quad m-1<\mu \leq m,
\end{array}\right.
$$

where $g$ is assumed to lie in $C_{-1}$ if $\mu \in \mathbb{N}_{0}$ or in $C_{-1}^{1}$ if $\mu \notin \mathbb{N}_{0}$. Then (8) has a unique solution in the space $C_{-1}^{m}$ of the form

$$
y(t)=\int_{0}^{t} s^{\mu-1} E_{\left(\mu-\mu_{1}, \ldots, \mu-\mu_{n}\right), \mu}\left(\lambda_{1} s^{\mu-\mu_{1}}, \ldots, \lambda_{1} s^{\mu-\mu_{n}}\right) g(t-s) d s+\sum_{k=0}^{m-1} c_{k} u_{k}(t), \quad t \geq 0,
$$

with, $k=0, \ldots, m-1$

$$
u_{k}(t)=\frac{t^{k}}{k !}+\sum_{i=l_{k}+1}^{n} \lambda_{i} t^{k+\mu-\mu_{i}} E_{\left(\mu-\mu_{1}, \ldots, \mu-\mu_{n}\right), k+1+\mu-\mu_{i}}\left(\lambda_{1} t^{\mu-\mu_{1}}, \ldots, \lambda_{n} t^{\mu-\mu_{n}}\right) .
$$

The natural numbers $l_{k}, k=0, \ldots, m-1$ are determined from the conditions $m_{l_{k}} \geq k+1$ and $m_{l_{k}+1} \leq k$. In the case $m_{i} \leq k, i=0, \ldots, m-1$, we set $l_{k}:=0$, and of $m_{i} \geq k+1, i=0, \ldots, m-1$, then $l_{k}:=n$.

\section{First and second fundamental solution of the time-fractional tele- graph equation with Laplace operator}

In this section we deduce the first and second FS of the multidimensional time-fractional telegraph equation with Laplace operator. We look for a function $G^{\alpha, \beta}(x, t)$ that satisfies the following Cauchy problem:

$$
\left\{\begin{array}{l}
\left(D_{t}^{\beta}+a D_{t}^{\alpha}-c^{2} \Delta_{x}\right) G^{\alpha, \beta}(x, t)=0 \\
G^{\alpha, \beta}(x, 0)=\delta(x) \\
\frac{\partial G^{\alpha, \beta}}{\partial t}(x, 0)=\delta(x)
\end{array}\right.
$$


where $x \in \mathbb{R}^{n}, t>0,1<\beta \leq 2,0<\alpha \leq 1, a \geq 0, c>0, \delta(x)=\prod_{j=1}^{n} \delta\left(x_{j}\right)$ is the distributional Dirac delta function in $\mathbb{R}^{n}$, and $\Delta_{x}$ is the Laplace operator in $\mathbb{R}^{n}$. Applying the Fourier transform in $\mathbb{R}^{n}$ to the Cauchy problem we get the following initial-value problem:

$$
\left\{\begin{array}{l}
\left(D_{t}^{\beta}+a D_{t}^{\alpha}+c^{2}|\kappa|^{2}\right) \widehat{G}^{\alpha, \beta}(\kappa, t)=0 \\
\widehat{G}^{\alpha, \beta}(\kappa, 0)=1 \\
\frac{\partial \widehat{G}^{\alpha, \beta}}{\partial t}(\kappa, 0)=1
\end{array} .\right.
$$

To solve the problem (10), we apply Theorem 2.8 with $\lambda_{1}=-a, \lambda_{2}=-c^{2}|\kappa|^{2}, \mu=\beta, \mu_{1}=\alpha, \mu_{2}=0, n=2$, $m=2, g(t)=0$, and $k=1$, obtaining the following solution:

$$
\widehat{G}^{\alpha, \beta}(\kappa, t)=\widehat{u}_{0}(\kappa, t)+\widehat{u}_{1}(\kappa, t),
$$

where

$$
\begin{aligned}
& \widehat{u}_{0}(\kappa, t)=1-c^{2}|\kappa|^{2} t^{\beta} E_{(\beta-\alpha, \beta), 1+\beta}\left(-a t^{\beta-\alpha},-c^{2}|\kappa|^{2} t^{\beta}\right), \\
& \widehat{u}_{1}(\kappa, t)=t-a t^{1+\beta-\alpha} E_{(\beta-\alpha, \beta), 2+\beta-\alpha}\left(-a t^{\beta-\alpha},-c^{2}|\kappa|^{2} t^{\beta}\right)-c^{2}|\kappa|^{2} t^{1+\beta} E_{(\beta-\alpha, \beta), 2+\beta}\left(-a t^{\beta-\alpha},-c^{2}|\kappa|^{2} t^{\beta}\right) .
\end{aligned}
$$

Taking into account Lemma 2.2 we have the following alternative representation of $\widehat{u}_{0}$ and $\widehat{u}_{1}$ :

$$
\begin{aligned}
& \widehat{u}_{0}(\kappa, t)=E_{(\beta-\alpha, \beta), 1}\left(-a t^{\beta-\alpha},-c^{2}|\kappa|^{2} t^{\beta}\right)+a t^{\beta-\alpha} E_{(\beta-\alpha, \beta), 1+\beta-\alpha}\left(-a t^{\beta-\alpha},-c^{2}|\kappa|^{2} t^{\beta}\right), \\
& \widehat{u}_{1}(\kappa, t)=t E_{(\beta-\alpha, \beta), 2}\left(-a t^{\beta-\alpha},-c^{2}|\kappa|^{2} t^{\beta}\right) .
\end{aligned}
$$

From (2) we have the following representation of $\widehat{u}_{0}$ and $\widehat{u}_{1}$ in the form of a double series:

$$
\begin{aligned}
\widehat{u}_{0}(\kappa, t)= & \sum_{p=0}^{+\infty} \sum_{q=0}^{+\infty} \frac{\Gamma(1+p+q)}{\Gamma(1+(\beta-\alpha) p+\beta q) p ! q !}\left(-a t^{\beta-\alpha}\right)^{p}\left(-c^{2}|\kappa|^{2} t^{\beta}\right)^{q} \\
& +a t^{\beta-\alpha} \sum_{p=0}^{+\infty} \sum_{q=0}^{+\infty} \frac{\Gamma(1+p+q)}{\Gamma(1+\beta-\alpha+(\beta-\alpha) p+\beta q) p ! q !}\left(-a t^{\beta-\alpha}\right)^{p}\left(-c^{2}|\kappa|^{2} t^{\beta}\right)^{q}, \\
\widehat{u}_{1}(\kappa, t)= & t \sum_{p=0}^{+\infty} \sum_{q=0}^{+\infty} \frac{\Gamma(1+p+q)}{\Gamma(2+(\beta-\alpha) p+\beta q) p ! q !}\left(-a t^{\beta-\alpha}\right)^{p}\left(-c^{2}|\kappa|^{2} t^{\beta}\right)^{q} .
\end{aligned}
$$

Moreover, from Lemma 2.4 and Corollary 2.5 it is also possible to obtain a representation of (12) and (13) in terms of double Mellin-Barnes integral and Fox H-function of two variables (due to the extension of the formulas we omit their presentation in the paper). Inverting the Fourier transform in (11) we get

$$
G^{\alpha, \beta}(x, t)=u_{0}(x, t)+u_{1}(x, t) .
$$

We will denote by $G_{1}^{\alpha, \beta}(x, t)=u_{0}(x, t)$ the first fundamental solution of the first equation of (9) that satisfies the initial conditions $G_{1}^{\alpha, \beta}(x, 0)=\delta(x)$ and $\frac{\partial G_{1}^{\alpha, \beta}}{\partial_{t}}(x, 0)=0$. Additionally, we denote by $G_{2}^{\alpha, \beta}(x, t)=u_{1}(x, t)$ the second fundamental solution of the first equation of (9) that satisfies the initial conditions $G_{2}^{\alpha, \beta}(x, 0)=0$ and $\frac{\partial G_{2}^{\alpha, \beta}}{\partial_{t}}(x, 0)=\delta(x)$. For obtaining the explicit expressions for $G_{1}^{\alpha, \beta}$ and $G_{2}^{\alpha, \beta}$ we recall the following formula (see [17])

$$
\frac{1}{(2 \pi)^{n}} \int_{\mathbb{R}^{n}} e^{-i \kappa \cdot x} \phi(|\kappa|) d \kappa=\frac{|x|^{1-\frac{n}{2}}}{(2 \pi)^{\frac{n}{2}}} \int_{0}^{+\infty} \phi(\tau) \tau^{\frac{n}{2}} J_{\frac{n}{2}-1}(\tau|x|) d \tau,
$$

where $J_{\nu}$ represents the Bessel function of first kind with index $\nu$, and the right hand side can be seen as a Hankel transform. We need also the following integral formula (see Formula (7) in [6, p. 22])

$$
\int_{0}^{+\infty} z^{\mu} J_{\nu}(z y)^{\frac{1}{2}} d z=\frac{2^{\mu+\frac{1}{2}}}{y^{\mu+1}} \frac{\Gamma\left(\frac{\mu}{2}+\frac{\nu}{2}+\frac{3}{4}\right)}{\Gamma\left(\frac{\nu}{2}-\frac{\mu}{2}+\frac{1}{4}\right)}
$$


which is valid under the condition $-\operatorname{Re}(\nu)-\frac{3}{2}<\operatorname{Re}(\mu)<-\frac{1}{2}$. Since the expressions for $G_{1}^{\alpha, \beta}$ are longer then the ones for $G_{2}^{\alpha, \beta}$, we are going to present only the calculations for $G_{2}^{\alpha, \beta}$. Applying the inverse Fourier transform to (13) and using (17), we get

$$
G_{2}^{\alpha, \beta}(x, t)=\frac{t|x|^{1-\frac{n}{2}}}{(2 \pi)^{\frac{n}{2}}} \int_{0}^{+\infty} \tau^{\frac{n}{2}} E_{(\beta-\alpha, \beta), 2}\left(-a t^{\beta-\alpha},-c^{2} \tau^{2} t^{\beta}\right) J_{\frac{n}{2}-1}(\tau|x|) d \tau .
$$

Making use of Lemma 2.4, and interchanging the integrals due to the convergence, we obtain

$$
\begin{aligned}
G_{2}^{\alpha, \beta}(x, t)=\frac{t|x|^{1-\frac{n}{2}}}{(2 \pi)^{\frac{n}{2}}} \frac{1}{(2 \pi i)^{2}} \int_{\mathcal{L}_{1}} \int_{\mathcal{L}_{2}} \frac{\Gamma(1+s+w) \Gamma(-s) \Gamma(-w)}{\Gamma(2+(\beta-\alpha) w+\beta s)} \\
\times\left(a t^{\beta-\alpha}\right)^{w}\left(c^{2} t^{\beta}\right)^{s} \int_{0}^{+\infty} \tau^{\frac{n}{2}+2 s} J_{\frac{n}{2}-1}(\tau|x|) d \tau d w d s .
\end{aligned}
$$

Finally, using (18) and Corollary 2.5, we obtain, under the condition $-\frac{n}{2}<\Re(s)<-\frac{n}{4}$, the representation of the second FS in terms of double Mellin-Barnes integral and H-function of two variables:

$$
\begin{aligned}
G_{2}^{\alpha, \beta}(x, t) & =\frac{t}{\pi^{\frac{n}{2}}|x|^{n}} \frac{1}{(2 \pi i)^{2}} \int_{\mathcal{L}_{1}} \int_{\mathcal{L}_{2}} \frac{\Gamma(1+s+w) \Gamma\left(\frac{n}{2}+s\right) \Gamma(-w)}{\Gamma(2+(\beta-\alpha) w+\beta s)}\left(a t^{\beta-\alpha}\right)^{w}\left(\frac{4 c^{2} t^{\beta}}{|x|^{2}}\right)^{s} d w d s \\
& =\frac{t}{\pi^{\frac{n}{2}}|x|^{n}} H_{1,1 ; 1,0 ; 0,1}^{0,1 ; 0,1 ; 1,0}\left[\begin{array}{c|c}
4 c^{2} t^{\beta}|x|^{-2} & (0 ; 1,1) ;\left(1-\frac{n}{2}, 1\right) ;- \\
a t^{\beta-\alpha} & (-1 ; \beta, \beta-\alpha) ;-;(0,1)
\end{array}\right],
\end{aligned}
$$

where the double Mellin-Barnes integral is convergent because the conditions (4) are fulfilled. For $G_{1}^{\alpha, \beta}$ we obtain, analogously, under the condition $-\frac{n}{2}<\Re(s)<-\frac{n}{4}$, the following representations for the first FS:

$$
\begin{aligned}
& G_{1}^{\alpha, \beta}(x, t)=\frac{|x|^{1-\frac{n}{2}}}{(2 \pi)^{\frac{n}{2}}} \int_{0}^{+\infty} \tau^{\frac{n}{2}} E_{(\beta-\alpha, \beta), 1}\left(-a t^{\beta-\alpha},-c^{2} \tau^{2} t^{\beta}\right) J_{\frac{n}{2}-1}(\tau|x|) d \tau \\
& +\frac{a t^{\beta-\alpha}|x|^{1-\frac{n}{2}}}{(2 \pi)^{\frac{n}{2}}} \int_{0}^{+\infty} \tau^{\frac{n}{2}} E_{(\beta-\alpha, \beta), 1+\beta-\alpha}\left(-a t^{\beta-\alpha},-c^{2} \tau^{2} t^{\beta}\right) J_{\frac{n}{2}-1}(\tau|x|) d \tau \\
& =\frac{1}{\pi^{\frac{n}{2}}|x|^{n}} H_{1,1 ; 1,0 ; 0,1}^{0,1 ; 0,1 ; 1,0}\left[\begin{array}{c|c}
4 c^{2} t^{\beta}|x|^{-2} & (0 ; 1,1) ;\left(1-\frac{n}{2}, 1\right) ;- \\
a t^{\beta-\alpha} & (0 ; \beta, \beta-\alpha) ;-;(0,1)
\end{array}\right] \\
& +\frac{a t^{\beta-\alpha}}{\pi^{\frac{n}{2}}|x|^{n}} H_{1,1 ; 1,0 ; 0,1}^{0,1 ; 0,1 ; 1,0}\left[\begin{array}{c|c}
4 c^{2} t^{\beta}|x|^{-2} & (0 ; 1,1) ;\left(1-\frac{n}{2}, 1\right) ;- \\
a t^{\beta-\alpha} & (-\beta+\alpha ; \beta, \beta-\alpha) ;-;(0,1)
\end{array}\right] .
\end{aligned}
$$

We end this section solving a fractional Cauchy-type problem.

Theorem 3.1 Let $x \in \mathbb{R}^{n}, t>0,1<\beta \leq 2,0<\alpha \leq 1, a \geq 0$, and $c>0$, then the fractional Cauchy type problem

$$
\left\{\begin{array}{l}
\left(D_{t}^{\beta}+a D_{t}^{\alpha}-c^{2} \Delta_{x}\right) h(x, t)=0 \\
h(x, 0)=f_{1}(x) \\
\frac{\partial h}{\partial t}(x, 0)=f_{2}(x)
\end{array}\right.
$$

is solvable, and its solution has the form

$$
h(x, t)=\int_{\mathbb{R}^{n}} G_{1}^{\alpha, \beta}(x-z, t) f_{1}(z) d z+\int_{\mathbb{R}^{n}} G_{2}^{\alpha, \beta}(x-z, t) f_{2}(z) d z,
$$

where $G_{1}^{\alpha, \beta}$ and $G_{2}^{\alpha, \beta}$ are the first and second fundamental solutions given by (24) and (22), respectively, and provided that the integrals in the right-hand side of (25) are convergent. 
Remark 3.2 In Theorem 3.1, when $a=0$ the solution (25) coincides with the one presented in [14, Cor.6.4]. Moreover, considering $a=0$ the first fundamental solution $G_{1}^{\alpha, \beta}$ coincides with the expression presented in [7,14] for the fundamental solution of the time-fractional diffusion-wave operator.

\section{Fundamental solution of the time-fractional equation with Dirac operator}

\subsection{Clifford analysis}

Now we recall some basic fact about Clifford analysis. Consider the $n$-dimensional vector space $\mathbb{R}^{n}$ endowed with an orthonormal basis $\left\{e_{1}, \cdots, e_{n}\right\}$. We define the universal real Clifford algebra $C \ell_{0, n}$ as the $2^{n}$-dimensional associative algebra which obeys the multiplication rule

$$
e_{i} e_{j}+e_{j} e_{i}=-2 \delta_{i, j}, \quad i, j=1, \ldots, n .
$$

A vector space basis for $C \ell_{0, n}$ is generated by the elements $e_{0}=1$ and $e_{A}=e_{h_{1}} \cdots e_{h_{k}}$, where $A=$ $\left\{h_{1}, \ldots, h_{k}\right\} \subset M=\{1, \ldots, n\}$, for $1 \leq h_{1}<\cdots<h_{k} \leq n$. Each element $x \in C \ell_{0, n}$ can be represented by $x=\sum_{A} x_{A} e_{A}$, with $x_{A} \in \mathbb{R}$. We introduce the complexified Clifford algebra $\mathbb{C}_{n}$ as the tensor product

$$
\mathbb{C}_{n}:=\mathbb{C} \otimes C \ell_{0, n}=\left\{w=\sum_{A} w_{A} e_{A}, w_{A} \in \mathbb{C}, A \subset M\right\},
$$

where the imaginary unit $i$ of $\mathbb{C}$ commutes with the basis elements, i.e., $i e_{j}=e_{j} i$ for all $j=1, \ldots, n$.

A $\mathbb{C}_{n}$-valued function defined on an open set $U \subseteq \mathbb{R}^{n}$ has the representation $f=\sum_{A} f_{A} e_{A}$ with $\mathbb{C}$ valued components $f_{A}$. Properties such as continuity and differentiability needs to be understood componentwisely. Next, we introduce the Euclidean Dirac operator $D_{x}=\sum_{j=1}^{n} e_{j} \partial_{x_{j}}$, which factorizes the $n$-dimensional Euclidean Laplacian, i.e., $D_{x}^{2}=-\Delta=-\sum_{j=1}^{n} \partial_{x_{j}}^{2}$. A Clifford valued $C^{1}$-function $f$ is called left-monogenic if it satisfies $D_{x} f=0$ on $U$ (resp. right-monogenic if it satisfies $f D_{x}=0$ on $U$ ).

In order to introduce a Witt basis to define a parabolic Dirac operator we embed $\mathbb{R}^{n}$ into $\mathbb{R}^{n+2}$ and consider two new elements $e_{+}$and $e_{-}$such that $e_{+}^{2}=+1, e_{-}^{2}=-1$, and $e_{+} e_{-}=-e_{-} e_{+}$. Moreover, $e_{+}$and $e_{-}$ anticommute with all the basis elements $e_{j}, j=1, \ldots, n$. Hence, $\left\{e_{1}, \ldots, e_{n}, e_{+}, e_{-}\right\}$spans $\mathbb{R}^{n+1,1}$. With the elements $e_{+}$and $e_{-}$we construct two nilpotent elements $\mathfrak{f}$ and $\mathfrak{f}^{\dagger}$ given by

$$
\mathfrak{f}=\frac{e_{+}-e_{-}}{2} \text { and } \mathfrak{f}^{\dagger}=\frac{e_{+}+e_{-}}{2} .
$$

These elements satisfy the following relations

$$
(\mathfrak{f})^{2}=\left(\mathfrak{f}^{+}\right)^{2}=0, \quad \mathfrak{f f}^{+}+\mathfrak{f}^{+} \mathfrak{f}=1, \quad \mathfrak{f} e_{j}+e_{j} \mathfrak{f}=\mathfrak{f}^{+} e_{j}+e_{j} \mathfrak{f}^{+}=0, j=1, \ldots, n .
$$

The extended basis $\left\{e_{1}, \ldots, e_{n}, \mathfrak{f}, \mathfrak{f}^{\dagger}\right\}$ allow us to define the telegraph Dirac operator as $D_{x, t}:=D_{x}+\mathfrak{f}\left(A \partial_{t}^{2}+B \partial_{t}\right)$ $+\mathfrak{f}^{\dagger}$, where $D_{x}$ stands for the Dirac operator in $\mathbb{R}^{n}$, and $A, B \in \mathbb{R}$ with $A \neq 0$. The operator $D_{x, t}$ acts on $\mathbb{C}_{n}$-valued functions defined on time dependent domains $\Omega \times I \subseteq \mathbb{R}^{n} \times \mathbb{R}^{+}$, i.e., functions in the variables $\left(x_{1}, x_{2}, \ldots, x_{n}, t\right)$ where $x_{j} \in \mathbb{R}$ for $j=1, \ldots, n$, and $t \in \mathbb{R}^{+}$. For the sake of readability, we abbreviate the space-time tuple $\left(x_{1}, x_{2}, \ldots, x_{n}, t\right)$ simply by $(x, t)$, assigning $x=x_{1} e_{1}+\cdots+x_{n} e_{n}$. For additional details about Clifford analysis and the Witt basis, we refer the interested reader for instance to $[4,5,12]$.

\subsection{First and second fundamental solutions}

In this section we compute the first and second FS for the time-fractional telegraph Dirac operator defined by

$$
D_{x, t}^{\alpha, \beta}:=c D_{x}+\mathfrak{f}\left(D_{t}^{\beta}+a D_{t}^{\alpha}\right)+\mathfrak{f}^{\dagger},
$$

where $D_{x}$ is the Dirac operator in $\mathbb{R}^{n}, a \geq 0, c>0, D_{t}^{\alpha}$ and $D_{t}^{\beta}$ are the Caputo fractional derivatives of order $\alpha \in] 0,1]$ and $\beta \in] 1,2]$, respectively (see (7)). This operator factorizes the time-fractional telegraph operator $-c^{2} \Delta_{x}+D_{t}^{\beta}+a D_{t}^{\alpha}($ see $[9])$

$$
\left(D_{x, t}^{\alpha, \beta}\right)^{2}=-c^{2} \Delta_{x}+D_{t}^{\beta}+a D_{t}^{\alpha}
$$


Applying the operator $D_{x, t}^{\alpha, \beta}$ to $G^{\alpha, \beta}$, we obtain the fundamental solution of $D_{x, t}^{\alpha, \beta}$ given by

$$
\mathcal{G}^{\alpha, \beta}(x, t)=D_{x, t}^{\alpha, \beta} G^{\alpha, \beta}(x, t)=\mathcal{G}_{1}^{\alpha, \beta}(x, t)+\mathcal{G}_{2}^{\alpha, \beta}(x, t),
$$

where $\mathcal{G}_{1}^{\alpha, \beta}$ and $\mathcal{G}_{2}^{\alpha, \beta}$ are the first and second FS of $D_{x, t}^{\alpha, \beta}$ :

$$
\begin{aligned}
& \mathcal{G}_{1}^{\alpha, \beta}(x, t)=c D_{x} G_{1}^{\alpha, \beta}(x, t)+\mathfrak{f}\left(D_{t}^{\beta}+a D_{t}^{\alpha}\right) G_{1}^{\alpha, \beta}(x, t)+\mathfrak{f}^{\dagger} G_{1}^{\alpha, \beta}(x, t) \\
& \mathcal{G}_{2}^{\alpha, \beta}(x, t)=c D_{x} G_{2}^{\alpha, \beta}(x, t)+\mathfrak{f}\left(D_{t}^{\beta}+a D_{t}^{\alpha}\right) G_{2}^{\alpha, \beta}(x, t)+\mathfrak{f}^{\dagger} G_{2}^{\alpha, \beta}(x, t) .
\end{aligned}
$$

Indeed, by the above factorization we have

$$
D_{x, t}^{\alpha, \beta} \mathcal{G}^{\alpha, \beta}(x, t)=\left(-c^{2} \Delta_{x}+D_{t}^{\beta}+a D_{t}^{\alpha}\right) G^{\alpha, \beta}(x, t)=0,
$$

which shows that $\mathcal{G}^{\alpha, \beta}$ is a FS of $D_{x, t}^{\alpha, \beta}$. To deduce an integral representation for $\mathcal{G}^{\alpha, \beta}$ we start recalling the following differential relation proved in [9]

$$
D_{x}\left[|x|^{1-\frac{n}{2}} J_{\frac{n}{2}-1}(\tau|x|)\right]=-\frac{\tau x}{|x|^{\frac{n}{2}}} J_{\frac{n}{2}}(\tau|x|) .
$$

Moreover, taking into account (7), together with the series expansion of the bivariate Mittag-Leffler function (2), we have the following differentiation formula, for $0<\gamma \leq 2, k_{1}, k_{2} \in \mathbb{R}$ :

$$
D_{t}^{\gamma}\left[t^{b-1} E_{\left(a_{1}, a_{2}\right), b}\left(k_{1} t^{a_{1}}, k_{2} t^{a_{2}}\right)\right]=t^{b-1-\gamma} E_{\left(a_{1}, a_{2}\right), b-\gamma}\left(k_{1} t^{a_{1}}, k_{2} t^{a_{2}}\right) .
$$

As it was done previously, due to the extension of the involved expressions, we will present only the calculations for $\mathcal{G}_{2}^{\alpha, \beta}$. However, we remark that for the terms involving $\mathcal{G}_{1}^{\alpha, \beta}$ we proceed in a similar way. Thereby, applying $D_{x, t}^{\alpha, \beta}$ to (19) and using the Leibniz rule for improper integrals together with relations (30) and (31), we get the following expressions for the vectorial and $\mathfrak{f}$ components of $\mathcal{G}_{2}^{\alpha, \beta}$ :

$$
\begin{aligned}
D_{x} G_{2}^{\alpha, \beta}(x, t)= & \frac{-c t x}{(2 \pi|x|)^{\frac{n}{2}}} \int_{0}^{+\infty} \tau^{\frac{n}{2}+1} E_{(\beta-\alpha, \beta), 2}\left(-a t^{\beta-\alpha},-c^{2} \tau^{2} t^{\beta}\right) J_{\frac{n}{2}}(\tau|x|) d \tau \\
\left(D_{t}^{\beta}+a D_{t}^{\alpha}\right) G_{2}^{\alpha, \beta}(x, t)= & \frac{t^{1-\beta}|x|^{1-\frac{n}{2}}}{(2 \pi)^{\frac{n}{2}}} \int_{0}^{+\infty} \tau^{\frac{n}{2}} E_{(\beta-\alpha, \beta), 2-\beta}\left(-a t^{\beta-\alpha},-c^{2} \tau^{2} t^{\beta}\right) J_{\frac{n}{2}-1}(\tau|x|) d \tau \\
& +a \frac{t^{1-\alpha}|x|^{1-\frac{n}{2}}}{(2 \pi)^{\frac{n}{2}}} \int_{0}^{+\infty} \tau^{\frac{n}{2}} E_{(\beta-\alpha, \beta), 2-\alpha}\left(-a t^{\beta-\alpha},-c^{2} \tau^{2} t^{\beta}\right) J_{\frac{n}{2}-1}(\tau|x|) d \tau .
\end{aligned}
$$

For the $\mathfrak{f}^{\dagger}$-component the correspondent expression is (19). Making use of Lemma 2.4, then interchanging the integrals due to the convergence, and finally using (18) and Corollary 2.5, leads to the following representations in terms of double Mellin-Barnes integral and H-function of two variables:

$$
\begin{aligned}
D_{x} G_{2}^{\alpha, \beta}(x, t) & =\frac{-2 c x t}{\pi^{\frac{n}{2}}|x|^{n+2}} \frac{1}{(2 \pi i)^{2}} \int_{\mathcal{L}_{1}} \int_{\mathcal{L}_{2}} \frac{\Gamma(1+s+w) \Gamma\left(2+\frac{n}{2}+s\right) \Gamma(-w)}{\Gamma(2+(\beta-\alpha) w+\beta s)}\left(a t^{\beta-\alpha}\right)^{w}\left(\frac{4 c^{2} t^{\beta}}{|x|^{2}}\right)^{s} d w d s \\
& =\frac{-2 c x t}{\pi^{\frac{n}{2}}|x|^{n+2}} H_{1,1 ; 1,0 ; 0,1}^{0,1 ; 0,1 ; 1,0}\left[\begin{array}{c|c}
4 c^{2} t^{\beta}|x|^{-2} & (0 ; 1,1) ;\left(-1-\frac{n}{2}, 1\right) ;- \\
a t^{\beta-\alpha} & (-1 ; \beta, \beta-\alpha) ;-;(0,1)
\end{array}\right] .
\end{aligned}
$$




$$
\begin{aligned}
& \left(D_{t}^{\beta}+a D_{t}^{\alpha}\right) G_{2}^{\alpha, \beta}(x, t)=\frac{1}{\pi^{\frac{n}{2}}|x|^{n} t^{\beta-1}} \frac{1}{(2 \pi i)^{2}} \int_{\mathcal{L}_{1}} \int_{\mathcal{L}_{2}} \frac{\Gamma(1+s+w) \Gamma\left(\frac{n}{2}+s\right) \Gamma(-w)}{\Gamma(2-\beta+(\beta-\alpha) w+\beta s)}\left(a t^{\beta-\alpha}\right)^{w}\left(\frac{4 c^{2} t^{\beta}}{|x|^{2}}\right)^{s} d w d s \\
& +a \frac{t^{1-\alpha}}{\pi^{\frac{n}{2}}|x|^{n}} \frac{1}{(2 \pi i)^{2}} \int_{\mathcal{L}_{1}} \int_{\mathcal{L}_{2}} \frac{\Gamma(1+s+w) \Gamma\left(\frac{n}{2}+s\right) \Gamma(-w)}{\Gamma(2-\alpha+(\beta-\alpha) w+\beta s)}\left(a t^{\beta-\alpha}\right)^{w}\left(\frac{4 c^{2} t^{\beta}}{|x|^{2}}\right)^{s} d w d s \\
& =\frac{1}{\pi^{\frac{n}{2}}|x|^{n} t^{\beta-1}} H_{1,1 ; 1,0 ; 0,1}^{0,1 ; 0,1 ; 1,0}\left[\begin{array}{c|c}
4 c^{2} t^{\beta}|x|^{-2} & (0 ; 1,1) ;\left(1-\frac{n}{2}, 1\right) ;- \\
a t^{\beta-\alpha} & (-1+\beta ; \beta, \beta-\alpha) ;-;(0,1)
\end{array}\right] \\
& +a \frac{t^{1-\alpha}}{\pi^{\frac{n}{2}}|x|^{n}} H_{1,1 ; 1,0 ; 0,1}^{0,1 ; 0,1 ; 1,0}\left[\begin{array}{c|r}
4 c^{2} t^{\beta}|x|^{-2} & (0 ; 1,1) ;\left(1-\frac{n}{2}, 1\right) ;- \\
a t^{\beta-\alpha} & (-1+\alpha ; \beta, \beta-\alpha) ;-;(0,1)
\end{array}\right] .
\end{aligned}
$$

In the previous expressions, the double Mellin-Barnes integrals are convergent because the conditions (4) are fulfilled. Analogously, for the vectorial and $\mathfrak{f}$ components of $\mathcal{G}_{1}^{\alpha, \beta}$ we have:

$$
\begin{aligned}
& D_{x} G_{1}^{\alpha, \beta}(x, t)=\frac{-c x}{(2 \pi|x|)^{\frac{n}{2}}} \int_{0}^{+\infty} \tau^{\frac{n}{2}+1} E_{(\beta-\alpha, \beta), 1}\left(-a t^{\beta-\alpha},-c^{2} \tau^{2} t^{\beta}\right) J_{\frac{n}{2}}(\tau|x|) d \tau \\
& -\frac{a c x t^{\beta-\alpha}}{(2 \pi|x|)^{\frac{n}{2}}} \int_{0}^{+\infty} \tau^{\frac{n}{2}+1} E_{(\beta-\alpha, \beta), 1+\beta-\alpha}\left(-a t^{\beta-\alpha},-c^{2} \tau^{2} t^{\beta}\right) J_{\frac{n}{2}}(\tau|x|) d \tau \\
& =\frac{-2 c x}{\pi^{\frac{n}{2}}|x|^{n+2}} H_{1,1 ; 1,0 ; 0,1}^{0,1 ; 0,1 ; 1,0}\left[\begin{array}{c|c}
4 c^{2} t^{\beta}|x|^{-2} & (0 ; 1,1) ;\left(-1-\frac{n}{2}, 1\right) ;- \\
a t^{\beta-\alpha} & (0 ; \beta, \beta-\alpha) ;-;(0,1)
\end{array}\right] \\
& -\frac{2 a c x t^{\beta-\alpha}}{\pi^{\frac{n}{2}}|x|^{n+2}} H_{1,1 ; 1,0 ; 0,1}^{0,1 ; 0,1 ; 1,0}\left[\begin{array}{c|c}
4 c^{2} t^{\beta}|x|^{-2} & (0 ; 1,1) ;\left(-1-\frac{n}{2}, 1\right) ;- \\
a t^{\beta-\alpha} & (-\beta+\alpha ; \beta, \beta-\alpha) ;-;(0,1)
\end{array}\right] \\
& \left(D_{t}^{\beta}+a D_{t}^{\alpha}\right) G_{1}^{\alpha, \beta}(x, t)=\frac{t^{-\beta}|x|^{1-\frac{n}{2}}}{(2 \pi)^{\frac{n}{2}}} \int_{0}^{+\infty} \tau^{\frac{n}{2}} E_{(\beta-\alpha, \beta), 1-\beta}\left(-a t^{\beta-\alpha},-c^{2} \tau^{2} t^{\beta}\right) J_{\frac{n}{2}-1}(\tau|x|) d \tau \\
& +\frac{(1+a) t^{-\alpha}|x|^{1-\frac{n}{2}}}{(2 \pi)^{\frac{n}{2}}} \int_{0}^{+\infty} \tau^{\frac{n}{2}} E_{(\beta-\alpha, \beta), 1-\alpha}\left(-a t^{\beta-\alpha},-c^{2} \tau^{2} t^{\beta}\right) J_{\frac{n}{2}-1}(\tau|x|) d \tau \\
& +\frac{a t^{\beta-2 \alpha}|x|^{1-\frac{n}{2}}}{(2 \pi)^{\frac{n}{2}}} \int_{0}^{+\infty} \tau^{\frac{n}{2}} E_{(\beta-\alpha, \beta), 1+\beta-2 \alpha}\left(-a t^{\beta-\alpha},-c^{2} \tau^{2} t^{\beta}\right) J_{\frac{n}{2}-1}(\tau|x|) d \tau \\
& =\frac{1}{\pi^{\frac{n}{2}}|x|^{n} t^{\beta}} H_{1,1 ; 1,0 ; 0,1}^{0,1 ; 0,1 ; 1,0}\left[\begin{array}{c|c}
4 c^{2} t^{\beta}|x|^{-2} & (0 ; 1,1) ;\left(-\frac{n}{2}, 1\right) ;- \\
a t^{\beta-\alpha} & (\beta ; \beta, \beta-\alpha) ;-;(0,1)
\end{array}\right] \\
& +\frac{1+a}{\pi^{\frac{n}{2}}|x|^{n} t^{\alpha}} H_{1,1 ; 1,0 ; 0,1}^{0,1 ; 0,1,1,0}\left[\begin{array}{c|c}
4 c^{2} t^{\beta}|x|^{-2} & (0 ; 1,1) ;\left(-\frac{n}{2}, 1\right) ;- \\
a t^{\beta-\alpha} & (\alpha ; \beta, \beta-\alpha) ;-;(0,1)
\end{array}\right] \\
& +\frac{a t^{\beta-2 \alpha}}{\pi^{\frac{n}{2}}|x|^{n}} H_{1,1 ; 1,0 ; 0,1}^{0,1 ; 0,1 ; 1,0}\left[\begin{array}{c|r}
4 c^{2} t^{\beta}|x|^{-2} & (0 ; 1,1) ;\left(-\frac{n}{2}, 1\right) ;- \\
a t^{\beta-\alpha} & (-\beta+2 \alpha ; \beta, \beta-\alpha) ;-;(0,1)
\end{array}\right] .
\end{aligned}
$$


For the $\mathfrak{f}^{\dagger}$-component of $\mathcal{G}_{1}^{\alpha, \beta}$ the representations are given by the expressions (23) and (24). We end this section presenting an immediate application.

Theorem 4.1 Let $x \in \mathbb{R}^{n}, t>0,1<\beta \leq 2,0<\alpha \leq 1$, a $\geq 0$, and $c>0$, then the fractional Cauchy type problem

$$
\left\{\begin{array}{l}
\left(c D_{x}+\mathfrak{f}\left(D_{t}^{\beta}+a D_{t}^{\alpha}\right)+\mathfrak{f}^{\dagger}\right) h(x, t)=0 \\
h(x, 0)=f_{1}(x) \\
\frac{\partial h}{\partial t}(x, 0)=f_{2}(x)
\end{array}\right.
$$

is solvable, and its solution has the form

$$
h(x, t)=\int_{\mathbb{R}^{n}} \mathcal{G}_{1}^{\alpha, \beta}(x-z, t) f_{1}(z) d z+\int_{\mathbb{R}^{n}} \mathcal{G}_{2}^{\alpha, \beta}(x-z, t) f_{2}(z) d z,
$$

with $\mathcal{G}_{1}^{\alpha, \beta}$ and $\mathcal{G}_{2}^{\alpha, \beta}$ are the first and second fundamental solutions in (28) and (29), and provided that the integrals in the right-hand side of (34) are convergent.

Remark 4.2 When $a=0$ the first fundamental solution $\mathcal{G}_{1}^{\alpha, \beta}$ coincides with the expression presented in [7] for the fundamental solution of the time-fractional parabolic Dirac operator.

Acknowledgement: The authors were supported by Portuguese funds through the CIDMA - Center for Research and Development in Mathematics and Applications, and the Portuguese Foundation for Science and Technology ("FCT-Fundaçâo para a Ciência e a Tecnologia"), within project UID/MAT/ 0416/2013.

N. Vieira is Auxiliar Researcher, under the FCT Researcher Program 2014 (Ref: IF/00271/2014).

\section{References}

[1] R.G. Buschman, H-functions of two variables III, Pure Appl. Math. Sci., 9, (1978), 13-18.

[2] R.G. Buschman, H-functions of two variables I, Indian J. Math., 20, (1978), 132-153.

[3] M. Caputo, Linear Models of Dissipation Whose Q Is Almost Frequency Independent-II, Geophys. J. R. Astron. Soc., 13-No.5, (1967), 529-539.

[4] P. Cerejeiras, U. Kähler and F. Sommen, Parabolic Dirac operators and the Navier-Stokes equations over timevarying domains, Math. Meth. in Appl. Sc., 28-No.14, (2005), 1715-1724.

[5] R. Delanghe, F. Sommen and V. Souček, Clifford algebras and spinor-valued functions. A function theory for the Dirac operator, Mathematics and its Applications-Vol.53, Kluwer Academic Publishers, Dordrecht etc., 1992.

[6] A. Erdélyi, W. Magnus, F. Oberhettinger and G. Tricomi, Tables of integral transforms-Vol.II, Bateman Manuscript Project, California Institute of Technology, McGraw-Hill Book Company, New York-Toronto-London, 1954.

[7] M. Ferreira and N. Vieira, Fundamental solutions of the time fractional diffusion-wave and parabolic Dirac operators, J. Math. Anal. Appl., 447-No.1, (2017), 329-353.

[8] M. Ferreira, M.M. Rodrigues and N. Vieira, Fundamental solution of the multi-dimensional time fractional telegraph equation, Fract. Calc. Appl. Anal., Fract. Calc. Appl. Anal., 20-No.4, (2017), 868-894.

[9] M. Ferreira, M.M. Rodrigues and N. Vieira, Fundamental solution of the time-fractional telegraph Dirac operator, Math. Meth. in Appl. Sc., 40-No.18, (2017), 7033-7050.

[10] M. Ferreira and N. Vieira, Eigenfunctions and fundamental solutions of the Caputo fractional Laplace and Dirac operators, Modern Trends in Hypercomplex Analysis, Trends in Mathematics Series, S. Bernstein, U. Khler, I. Sabadini, F.Sommen (Eds.), (2016), 191-202.

[11] M. Ferreira, and N. Vieira, Eigenfunctions and fundamental solutions of the fractional Laplace and Dirac operators: the Riemann-Liouville case, Complex Anal. Oper. Theory, 10-No.5, (2016), 1081-1100.

[12] K. Gürlebeck and W. Sprößig, Quaternionic and Clifford calculus for physicists and engineers, Mathematical Methods in Practice, Wiley, Chichester, 1997.

[13] H.J. Haubold, A.M. Mathai and R.K. Saxena, Mittag-Leffler functions and their applications, J. Appl. Math., (2011), Article ID 298628, 51 p. 
[14] A. Kilbas, H.M. Srivastava and J.J. Trujillo, Theory and applications of fractional differential equations, NorthHolland Mathematics Studies-Vol.204, Elsevier, Amsterdam, 2006.

[15] Y. Luchko and R. Gorenflo, An operational method for solving fractional differential equations with the Caputo derivatives Acta Math. Vietnam., 24-No.2, (1999), 207-233.

[16] I. Podlubny, Fractional Differential Equations. An introduction to fractional derivatives, fractional differential equations, to methods of their solution and some of their applications, Mathematics in Science and Engineering 198, Academic Press, San Diego, 1999.

[17] S.G. Samko, A.A. Kilbas and O.I. Marichev, Fractional integrals and derivatives: theory and applications, Gordon and Breach, New York, NY, 1993.

[18] R.C. Tautz and I. Lerche, Application of the three-dimensional telegraph equation to cosmic-ray transport, Res. Astron. Astrophys., 16(10) (2016), 162. 